\title{
Terpene-Containing PEGylated Liposomes as Transdermal Carriers of a Hydrophilic Compound
}

\author{
Worranan Rangsimawong, Praneet Opanasopit, Theerasak Rojanarata, and \\ Tanasait Ngawhirunpat* \\ Faculty of Pharmacy, Silpakorn University; Nakhon Pathom 73000, Thailand. \\ Received July 24, 2014; accepted September 29, 2014; advance publication released online October 8, 2014
}

We investigated the effect of PEGylated liposomes (PLs) containing a terpene on the penetration of a hydrophilic compound through porcine skin. PLs composed of $\mathrm{N}$-(carbonyl-methoxypolyethyleneglycol2000)-1,2-distearoyl-sn-glycero-3-phosphoethanolamine ( PEG $\left._{2000}-\mathrm{DSPE}\right)$, the sodium salt of PEG 2000 $_{20 S P E}-$ phosphatidylcholine (PC), cholesterol (Chol), Tween 20, and $d$-limonene were prepared as carriers for fluorescein sodium (NaFI). The physicochemical characteristics of PLs and their effects on in vitro skin penetration were evaluated. Tape stripping was used to evaluate NaFI deposition in skin layers, and confocal laser scanning microscopy (CLSM) was used to investigate the depth of skin penetration and the pathways used by NaFI-loaded vesicles. PLs containing $d$-limonene were smaller and conferred higher entrapment efficiency and skin penetration on NaFI than did PLs and conventional liposomes (CLs). The deposition of NaFI from PLs with $d$-limonene was greater in epidermis and dermis $(6.10 \pm 1.74 \mu \mathrm{g})$ than stratum corneum $(2.06 \pm 0.47 \mu \mathrm{g})$. CLSM images revealed that NaFI penetrated into the deepest skin layer with maximum fluorescence intensity. NaFI penetrated deeper $(180 \mu \mathrm{m})$ in follicular than nonfollicular regions $(145 \mu \mathrm{m})$, suggesting a transfollicular pathway predominates in skin penetration by NaFI-loaded PLs. In conclusion, grafting PEG onto ultra-deformable liposomes may enhance transdermal NaFI delivery and may be used as a carrier to prolong liposome circulation time.

Key words polyethylene glycol (PEG)ylated liposome; hydrophilic compound; skin permeation; confocal laser scanning microscopy

Liposomes have been used for dermal and transdermal drug delivery. Lipids, usually phospholipids arranged in one or more concentric bilayers enclosing an equal number of aqueous compartments, can entrap hydrophilic agents within the inner aqueous sphere. Lipophilic agents can intercalate into the lipid bilayer. ${ }^{1)}$ However, traditional liposomes are of little or no value for transdermal drug delivery because they do not deeply penetrate skin, remaining confined to the upper layers of the stratum corneum. ${ }^{2)}$ Cevc et al. have reported deformable liposomes (Transfersomes ${ }^{\circledR}$ ), the first generation of elastic vesicles that can increase skin permeability, but only when applied under non-occluded conditions. ${ }^{3)}$ They consist of phospholipids and an edge activator. An edge activator is often a single-chain surfactant that destabilizes the lipid bilayer of the vesicles and increases deformability. ${ }^{4}$

Terpenes have been widely used as skin penetration enhancers for both hydrophilic and hydrophobic drugs. Monoterpenes such as $d$-limonene $\left(\mathrm{C}_{10} \mathrm{H}_{16}\right)$ were generally more efficacious due to their small molecular sizes. $d$-Limonene fluidizes or perturbs the integrity of the barrier function of stratum corneum for enhanced the transport of drugs through skin. ${ }^{5)}$ Ultra-deformable liposomes containing Tween 20 as an edge activator and terpenes as skin penetration enhancers have been reported to enhance skin penetration of NaFI by a synergistic effect of penetration enhancer, using mainly a transfollicular pathway, with smaller contributions from intercellular and transcellular pathways. ${ }^{6}$

A stealth liposome strategy can be achieved by modifying the surface of liposomal membranes with hydrophilic polymer conjugates, such as poly(ethylene glycol) (PEG). PEG is the most widely used polymer conjugate and reduces systemic

The authors declare no conflict of interest

* To whom correspondence should be addressed. e-mail: tanasait@su.ac.th uptake of mononuclear phagocytes. PEGylated liposomes (PLs) were developed to improve the blood circulation time of liposomes after intravenous administration. PEG has been widely used as a polymeric stabilizer that can be incorporated into the liposome surface in difference ways. The most widely used method at present is to anchor the polymer in the liposome membrane via a cross-linked lipid (i.e., PEG-distearoylphosphatidylethanolamine (PEG-DSPE)). ${ }^{6}$ In addition, it is possible to improve the colloidal stability of liposomes by changing the physicochemical properties of the particles via the polymer coating. Shielding the liposomes with a hydrophilic polymer coating (PEG) causes repulsion between bilayers, and thereby reduces the interactions between liposomes in the dispersion. ${ }^{7)}$

Few reports are available on dermal application of PLs. Jain et al. reported the topical application of PEGylated surfactantcontaining liposomes: PEGylation of surfactant-containing liposomes can increase the skin permeation of the low molecular weight drug, Zidovudine, by binding to water molecules, which could increase the hydration of the stratum corneum resulting in enhanced permeation of the stratum corneum barrier. $^{8)}$ In addition, calcipotriol-loaded liposomes with $1 \mathrm{~mol} \%$ PEG significantly increased the accumulation of calcipotriol in skin and hair follicles compared to non-PEGylated liposomes. The size of the liposomes affected the penetration of calcipotriol into the stratum corneum: small unilamellar vesicles enhanced calcipotriol penetration more than large ones. ${ }^{9)}$ The use of PLs as carriers for transdermal drug delivery requires further study. Liposomes with long circulation times containing skin penetration enhancers may yield a transdermal drug delivery system that can affect the blood circulation time of liposomes.

Fluorescein sodium $(\mathrm{NaFI})$ is a low molecular hydrophilic 
compound (molecular weight (MW) 376 Da, $\log P=-1.52$ ) that is used as a diagnostic tool in topical application. ${ }^{10)} \mathrm{Hy}-$ drophilic compound shows low skin permeation because of its physicochemical property. The stratum corneum is also known to exhibit selective permeability with respect to the type of diffusing molecules, including hydrophilicity, size, and hydrogen-bonding ability. ${ }^{11)}$ Therefore, the aim of this study was to investigate the effect of PLs on the penetration of a hydrophilic compound into porcine skin. NaFI, a fluorescent hydrophilic compound, was entrapped in vesicles. PLs were prepared by sonication. Particle size, shape, zeta potential, entrapment efficiency $(\% \mathrm{EE})$, and in vitro skin penetration were investigated. Tape stripping and confocal laser scanning microscopy (CLSM) were also used to investigate the penetration pathways of the vesicles and their skin penetration depths.

\section{MATERIALS AND METHODS}

Materials Egg phosphatidylcholine (PC) and the $\mathrm{Na}$ salt of $N$-(carbonyl-methoxypolyethyleneglycol-2000)-1,2-distearoyl-sn-glycero-3-phosphoethanolamine $\left(\mathrm{PEG}_{2000}-\mathrm{DSPE}\right)$ were purchased from Lipoid GmbH, Ludwigshafen, Germany. Cholesterol (Chol) was purchased from Carlo Erba Reagent, Ronado, Italy. Tween 20 was purchased from Ajax Finechem, Auckland, New Zealand. Fluorescein sodium (NaFI) and $d$ limonene were purchased from Sigma-Aldrich, St. Louis, MO, U.S.A.

Liposome Preparation Liposomes were prepared by sonication. Liposome composition [phospholipid (PC), $\mathrm{PEG}_{2000}$ DSPE, Chol, Tween 20 and $d$-limonene] is shown in Table 1. A mixture of PC, Chol, and $\mathrm{PEG}_{2000}-\mathrm{DSPE}$ dissolved in chloroform-methanol $(2: 1, \mathrm{v} / \mathrm{v})$ was added to a test tube. Rh-PE was added to the mixture as a fluorescence probe. The molar ratio of PC to Rh-PE was 100:1. The solvent was evaporated using a stream of nitrogen. The lipid film was placed in a desiccator until completely dry (6h). NaFI was dissolved in phosphate-buffered saline (PBS; pH 7.4) at $0.21 \%(\mathrm{w} / \mathrm{v})$ and added to the lipid film to give hydrated liposome vesicles. For PLs with $d$-limonene, the dispersion was mixed with Tween 20 and $d$-limonene at $2 \%(\mathrm{w} / \mathrm{v})$ and $1 \%(\mathrm{w} / \mathrm{v})$, respectively. All formulations were probe-sonicated for $30 \mathrm{~min}$ to reduce the size of the liposomes. ${ }^{12)}$

Characterization of PEGylated Liposomes: Particle Size and Surface Charge Each liposome formulation was diluted with an appropriate amount of water and measured for size distribution and zeta potential, using a Dynamic Light Scattering (DLS) particle size analyzer (Zetasizer Nano-ZS, Malvern Instrument, Worcestershire, U.K.) with a $4 \mathrm{~mW} \mathrm{He}-\mathrm{Ne}$ laser at a scattering angle of $173^{\circ}$. All measurements were carried out under ambient conditions in triplicate.

Atomic Force Microscopy (AFM) Atomic force microscopy (SPA400, SPI4000, SII Seiko Nanotechnology, Tokyo,
Japan) operating in tapping mode at room temperature $\left(25^{\circ} \mathrm{C}\right)$ was used to determine the morphology and structure of liposomal formulation. Liposomal formulations were diluted with distilled water before dropped onto a mica sheet. The cantilever tip had a resonant frequency $\pm 10 \%, \mathrm{kHz}=210$ and force constant $\pm 20 \%, \mathrm{~N} / \mathrm{m}=6.1$ (NT-MDT, Moscow, Russia). Scanning area was $1 \mu \mathrm{m}$ or $2.5 \mu \mathrm{m}$ square and scan rate was around $1.0 \mathrm{~Hz}$. The AFM tapping mode data were analyzed using SPI4000 version 4.17E (SII Seiko Nanotechnology).

Drug Entrapment Efficiency (\%EE) The liposome dispersion $(0.5 \mathrm{~mL})$ was placed in an ultrafiltration tube with a molecular weight cutoff of $3000 \mathrm{Da}$ (Microcon YM-3; Minipore, Billerica, MA, U.S.A.) and centrifuged at $4^{\circ} \mathrm{C}$ at 10000 $\times \boldsymbol{g}$ for $60 \mathrm{~min}$. The filtrate was discarded, and $0.25 \mathrm{~mL}$ of PBS was added to the retentate before further centrifugation at $4^{\circ} \mathrm{C}$ at $10000 \times \boldsymbol{g}$ for $40 \mathrm{~min}$. The collected NaFI-loaded liposomes in the retentate were then disrupted with $0.2 \mathrm{~mL}$ of $0.1 \%(\mathrm{w} / \mathrm{v})$ Triton X-100 and centrifuged at $4^{\circ} \mathrm{C}$ at $10000 \times \boldsymbol{g}$ for $10 \mathrm{~min}$. The NaFI content of the supernatant was determined by fluorescence analysis. The drug \%EE was calculated with the following:

$$
\% \mathrm{EE}=\left(C_{\mathrm{L}} / C_{\mathrm{i}}\right) \times 100
$$

Eq. 1

where $C_{\mathrm{L}}$ is the concentration of NaFI in the liposomal formulation, and $C_{\mathrm{i}}$ is the initial concentration of NaFI added. ${ }^{12)}$

In Vitro Skin Penetration Study: Preparation of the Porcine Skin Abdominal porcine skin was taken from intrapartum stillborn animals from a farm in Nakhon Pathom. Subcutaneous fat was carefully removed using medical scissors and surgical blades. The skin thickness was approximately $0.6-0.7 \mathrm{~mm}$. Samples were frozen at $-20^{\circ} \mathrm{C}$ until use and thawed at room temperature in PBS prior to experiments.

Skin Penetration In vitro permeation studies with porcine abdominal skin were carried out following Subongkot et $a l .{ }^{6)}$ Permeation of NaFI through porcine skin was performed using Franz diffusion cells. Briefly, approximately $2 \mathrm{~mL}$ of NaFI-loaded liposome solution was applied to the skin in the donor compartment. The receptor compartment of the cell was filled with $6 \mathrm{~mL}$ of phosphate-buffered saline (PBS) $\mathrm{pH}$ 7.4. Diffusion continued for $24 \mathrm{~h}$. Samples were taken at predetermined times $(1,2,4,6,8$, and $24 \mathrm{~h}) ; 0.5 \mathrm{~mL}$ of receiver medium was withdrawn for analysis using fluorescence detection, and the same volume of PBS was added to the receiver compartment to maintain a constant volume. Each sample was analyzed in triplicate.

In Vitro Tape Stripping After the in vitro permeation studies (at $8 \mathrm{~h}$ ), tape stripping was performed to collect the stratum corneum for each formulation. The skin was washed with PBS and blotted dry with soft tissue. Visible hair was removed with scissors. Transparent adhesive tape (3M, Hutchinson, MA, U.S.A.) was pressed onto the skin $\left(2.31 \mathrm{~cm}^{2}\right)$

Table 1. Lipid Compositions of the Different Liposomal Formulations

\begin{tabular}{lccc}
\hline \hline Formulations & PC:Chol: PEG $_{2000}-\mathrm{DSPE}(\mathrm{mm})$ & Tween20 (mM) & $d$-Limonene (mM) \\
\hline Conventional liposomes (CLs) & $10: 2$ & - & - \\
PEGylated liposomes (PLs) & $10: 2: 0.12$ & - & - \\
PLs with Tween20 & $10: 2: 0.12$ & 16.29 & - \\
PLs with $d$-limonene & $10: 2: 0.12$ & 16.29 & 73.41 \\
\hline
\end{tabular}


with the thumb using a vinyl glove. Approximately 20 strips were applied, quickly removed and collected. After stripping, the skin was cut into small pieces. The collected stratum corneum and remaining skin (epidermis and dermis (ED)) were added to $20 \mathrm{~mL}$ of PBS, probe-sonicated for $20 \mathrm{~min}$, and then centrifuged for $10 \mathrm{~min}$ at $12000 \mathrm{rpm} .{ }^{13,14)}$ The NaFI-containing stratum corneum and ED were analyzed using fluorescence detection. Each sample was analyzed in triplicate. ${ }^{15)}$

Fluorescence Analysis The NaFI concentration was analyzed using a fluorescence spectrophotometer (Fusion $^{\mathrm{TM}}$ Universal Microplate Analyzer, Packard Instrument Co., Inc., Downers Grove, IL, U.S.A.). The excitation wavelength was $485 \mathrm{~nm}$, and the emission wavelength was $535 \mathrm{~nm}$. Samples $(100 \mu \mathrm{L})$ were pipetted into a black 96-well plate, and fluorescence was determined in triplicate.

CLSM Study After in vitro skin penetration at $4 \mathrm{~h}$, the depth of the NaFI penetration through the skin was visualized as previously reported. ${ }^{12)}$ The $x-z$ sectioning confocal images were obtained using the $\times 20$ objective lens system of an inverted Zeiss LSM 510 META microscope (Carl Zeiss, Jena, Germany) equipped with $\mathrm{He}-\mathrm{Ne}$ (excitation wavelength $543 \mathrm{~nm}$; emission wavelength $580 \mathrm{~nm}$ ), Ar (excitation $488 \mathrm{~nm}$; emission $514 \mathrm{~nm}$ ) and diode lasers (excitation $358 \mathrm{~nm}$; emission $461 \mathrm{~nm}$ ). A piece of tissue was placed on a coverslip $(22 \times 50 \mathrm{~mm})$ with the stratum corneum facing up toward the microscope condenser. Sufficient methyl salicylate was added to immerse the tissue. Confocal images are illustrated as $x-z$ axis serial optical sections. Fluorescence intensity was evaluated at the middle horizontal line of each image using Zeiss LSM 5 operating software. Mean fluorescence intensity was plotted against skin depth.

Data Analysis Cumulative amount was plotted against time and steady-state flux determined as the slope of the linear portion of the plot. Lag time was obtained by extrapolating the linear portion of the penetration profile to the abscissa. The enhancement ratio (ER) was calculated with the following equation:

$$
\begin{array}{cc}
\mathrm{ER}^{1}=\frac{\text { Flux of PLs formulations }}{\text { Flux of CLs }} & \text { Eq. } 2 \\
\mathrm{ER}^{2}=\frac{\text { Flux of PLs with Tween } 20 \text { or } d \text {-limonene }}{\text { Flux of PLs }} & \text { Eq. } 3
\end{array}
$$

All experimental measurements were collected in triplicate. The values are expressed as the mean and standard deviation (S.D.). Statistical significance was analyzed using KruskalWallis one-way ANOVA followed by Tukey's post hoc test. The significance level was set at $p<0.05$.

\section{RESULTS AND DISCUSSION}

Physicochemical Characteristics The physicochemical characteristics of different liposome formulations are presented in Table 2. The average particle size of liposome formulations was in the following order: PLs with Tween 20 $(26.88 \pm 0.54 \mathrm{~nm})<$ PLs with $d$-limonene $(31.46 \pm 0.28 \mathrm{~nm})<$ PLs $(68.82 \pm 0.62 \mathrm{~nm})<$ CLs $(106.43 \pm 0.42 \mathrm{~nm})$. The particle size of PLs was smaller than that of CLs because PEG molecules at the surface of liposomes strongly reduce attractive forces (van der Waals) and increase repulsive forces (steric, electrostatic, and hydration). ${ }^{16)}$ PLs with Tween 20 or $d$-limonene were significantly smaller than other formulations, indicating that the particle size of PLs was affected by Tween 20. ${ }^{12)}$ All formulations showed a polydispersity index (PDI) below 0.3 , indicating a narrow size distribution. The zeta potential of all formulations exhibited negative surface charge $(-8.74$ to $-10.25 \mathrm{mV}$ ) due to the presence of zwitterionic compounds. The $\mathrm{pH}$ of the liposome formulations used (7.4) was higher than the isoelectric point $(\mathrm{p} I)$ of $\mathrm{PC}: 6$ to $6.7{ }^{17)} \mathrm{PC}$ vesicles had an overall negative charge under these conditions. The negative charge of PEG-DSPE also increases the repulsive forces on the liposome surface, which may improve the colloidal stability of liposomes. ${ }^{16)}$

The AFM images of liposomal formulation are shown in Fig. 1. The liposomal morphology (shape, structure, surface morphology and size measurement) was obtained using AFM. $^{18)}$ All formulations were spherical structure of the small unilamellar vesicles. The geometric mean diameter of CLs, SLs, SLs with Tween20, and SLs with $d$-limonene were $132.35 \pm 1.97 \mathrm{~nm}, 45.67 \pm 4.31 \mathrm{~nm}, 30.11 \pm 8.39 \mathrm{~nm}$, and $31.36 \pm 5.09 \mathrm{~nm}$, respectively. The height of CLs, SLs, SLs with Tween20, and SLs with $d$-limonene were $27.40 \pm 1.97 \mathrm{~nm}$, $9.70 \pm 0.91 \mathrm{~nm}, 6.53 \pm 2.23 \mathrm{~nm}$, and $6.28 \pm 1.67 \mathrm{~nm}$, respectively. The diameter in each liposome was higher than the height values because of the flattening of vesicles. The shape of liposomes can change after deposited on mica support. The deformation of liposomes was induced by the interaction between the liposome vesicle and the support, and the continuous movement of the tip that depended on the vesicle composition. $^{19)}$

The drug entrapment efficiency (\%EE) of liposome formulations was in the following order: PLs with Tween20>PLs with $d$-limonene $>$ PLs $>$ CLs. This indicates that NaFI (a hydrophilic compound) can be entrapped in the aqueous compartment of liposome vesicles. The membrane bilayer became more polar in the presence of PEG-lipids, resulting in increased efficiency of incorporation of the hydrophilic drug. ${ }^{13)}$ In addition, an increase in the content of edge activator may have led to formation of pores in the bilayer. ${ }^{20)}$ Edge activators and terpenes destabilize the lipid bilayers of vesicles and

Table 2. Characterization Parameters of the Different Liposomal Formulations

\begin{tabular}{lcccc}
\hline \hline Formulations & Particle size $(\mathrm{nm})$ & PDI & Zeta potential $(\mathrm{mV})$ & \%EE \\
\hline Conventional liposomes (CLs) & $106.43 \pm 0.42$ & $0.25 \pm 0.42$ & $-9.06 \pm 0.58$ & $17.08 \pm 0.64$ \\
PEGylated liposomes (PLs) & $68.82 \pm 0.62$ & $0.22 \pm 0.01$ & $-8.74 \pm 0.22$ & $21.24 \pm 1.42$ \\
PLs with Tween20 & $26.88 \pm 0.54$ & $0.21 \pm 0.02$ & $-10.97 \pm 0.80$ & $34.72 \pm 6.41$ \\
PLs with $d$-limonene & $31.46 \pm 0.28$ & $0.21 \pm 0.02$ & $-10.25 \pm 1.78$ & $30.30 \pm 0.71$ \\
\hline
\end{tabular}

Each value represents the mean \pm S.D. $(n=3)$ 


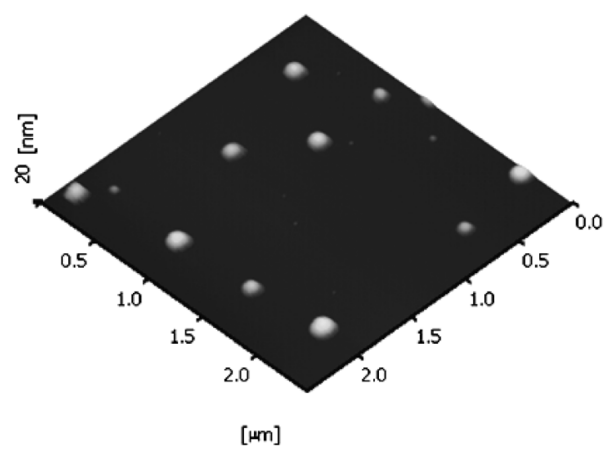

(A)

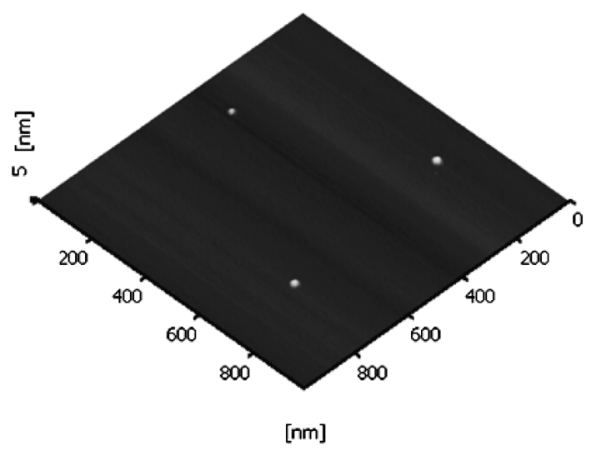

(C)

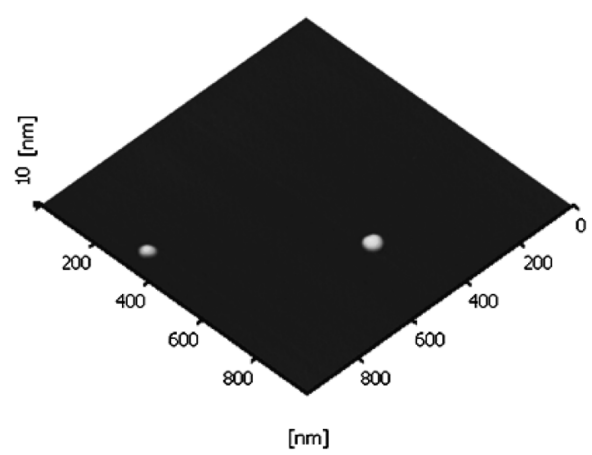

(B)

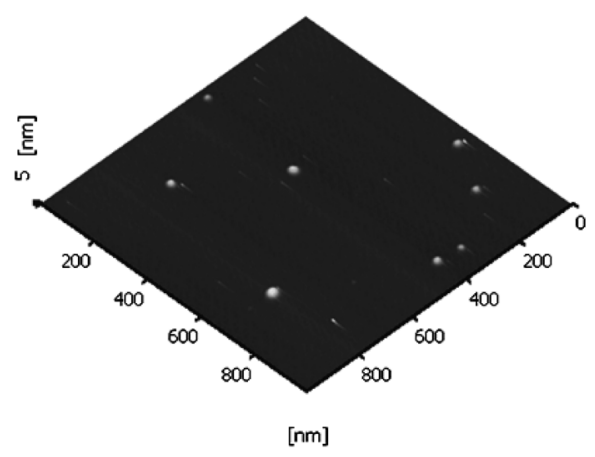

(D)

Fig. 1. Atomic Force Microscopy (AMF) Images of (A) CLs, (B) PLs, (C) PLs with Tween20, and (D) PLs with $d$-Limonene

Table 3. The Penetration Parameters of NaFI from Liposome Formulations

\begin{tabular}{lcccc}
\hline \hline Formulation & Flux $\left(\mu \mathrm{g} / \mathrm{cm}^{2} / \mathrm{h}\right)$ & Lag time $(\mathrm{h})$ & $\mathrm{ER}^{1}$ & $\mathrm{ER}^{2}$ \\
\hline NaFI solution (Sol) & $0.0058 \pm 0.00$ & $0.54 \pm 0.19$ & - & - \\
CLs & $0.0078 \pm 0.01$ & $1.00 \pm 0.35$ & - & - \\
PLs & $0.0639 \pm 0.05^{*}$ & $0.86 \pm 0.00$ & 17.24 & - \\
PLs with Tween20 & $0.1345 \pm 0.14^{*}$ & $0.71 \pm 0.14$ & 68.28 \\
PLs with $d$-limonene & $0.5326 \pm 0.11^{*}$ & $0.48 \pm 0.16$ & 8.10 \\
\hline
\end{tabular}

Each value represents the mean \pm S.D. $(n=3) . * p<0.05$.

increase the flexibility of the membrane., ${ }^{4,21}$

In Vitro Skin Penetration The amount of NaFI delivered from different liposome formulations into the skin plotted against time is shown in Fig. 2. The cumulative NaFI permeation from PLs was higher than from CLs or the NaFI solution (Sol), indicating that a hydrophilic polymer lipid $\left(\mathrm{PEG}_{2000}-\right.$ DSPE) increases skin permeation by NaFI. The penetration parameters of NaFI from liposome formulations are shown in Table 3. The flux of PLs with $d$-limonene, PLs with Tween 20 and PLs was significantly higher than CLs and NaFI sol. Compared with CLs, PLs with $d$-limonene had the highest transdermal flux (68.28-fold), followed by PLs with Tween 20 (17.24-fold) and PLs (8.20-fold), respectively. The flux of CLs incorporated with Tween 20 or $d$-limonene was not significantly different compared with PLs with Tween 20 or $d$-limonene. These results indicated that the pegylation had no effect in skin penetration enhancement in liposomes incorporated with Tween 20 or $d$-limonene, however however it significantly affected in conventional liposomes. $\mathrm{PEG}_{2000}$-DSPE gave higher skin penetration at 1 than 0.5 or $5 \mathrm{~mol} \%$ lipid. The conformation of the PEG polymer grafted onto the liposome surface is dependent on PEG concentration. In liposomes containing up to $4 \mathrm{~mol} \%$ grafted PEG (mushroom regime), neighboring coils did not interact laterally. In grafted PEG concentrations above $4 \mathrm{~mol} \%$, neighboring PEG chains push against each other, leading to a brush regime. ${ }^{22)}$ According to previous reports, calcipotriol-loaded liposomes with $1 \mathrm{~mol} \%$ PEG-DSPE significantly increased accumulation of calcipotriol in the stratum corneum compared to formulations with $0,0.5$, and $5 \mathrm{~mol} \%$. PEG (hydrophilic polymer) may increase skin penetration by binding to water molecules, increasing the hydration of the stratum corneum and enhancing permeation of this barrier. ${ }^{9)}$

PLs with $d$-limonene gave the highest skin permeation. For $\mathrm{ER}^{2}$, PLs with $d$-limonene was 8.33 -fold, whereas the $\mathrm{ER}^{2}$ of PLs with Tween 20 was 2.10 -fold increase in flux compared to PLs. These results indicated that the incorporation of Tween 20 and $d$-limonene into liposomes resulted in a synergistic enhancement of skin penetration of NaFI. ${ }^{5}$ The addition of Tween20 and $d$-limonene to CLs increased the fluidity of vesicle layers near the hydrophobic phospholipid acryl chains and enhanced NaFI penetration though porcine skin. ${ }^{12,15)} d$ Limonene is a terpene, which have been used extensively for topical delivery of both hydrophilic and lipophilic drugs. When the skin is treated with terpenes, the network of hydrogen bonds between ceramides may be broken, which may modify the organization of lipids in the stratum corneum. ${ }^{23,24)}$ 
Tween 20 is an edge activator providing the greatest deformability of neutral or negatively charged conventional liposomes. $\left.{ }^{25}\right)$ Thus, the addition of Tween 20 and $d$-limonene to PLs enhances the NaFI penetration of porcine skin. Moreover, this result may be due to the size of the liposomes ${ }^{14)}$ : small PLs gave higher skin penetration of NaFI than did larger ones.

In Vitro Tape Stripping Tape stripping is used to quantify drug penetration through skin and to examine the distribution of substances within the stratum corneum. ${ }^{26)}$ As shown in Fig. 3, the amount of NaFI in the stratum corneum was $3.24 \pm 0.45 \mu \mathrm{g}, 3.43 \pm 0.12 \mu \mathrm{g}, 2.22 \pm 0.35 \mu \mathrm{g}$, and $2.06 \pm 0.47 \mu \mathrm{g}$ for CLs, PLs, PLs with Tween20, and PLs with $d$-limonene, respectively. The NaFI penetration of the stratum corneum from CLs and PLs was significantly higher than from other formulations $(p<0.05)$. Traditional liposomes containing phospholipids and cholesterol do not penetrate skin deeply but rather remain confined to the upper layers of the stratum corneum. ${ }^{2)}$ The modification of the liposome structure by surface modification with PEG-lipids significantly enhanced NaFI delivery into deep skin layers compared to conventional liposomes. PEG is a hydrophilic polymer conjugated with phospholipids, which leads to better hydration of the stratum corneum than do phospholipids without PEG. ${ }^{27)}$ However, there was no significant difference between PLs and CLs (Fig. 3).

Several procedures are used to produce liposomes for the delivery of molecules into the deeper layers of the skin, showing better stability, higher drug entrapment efficiency or simply modulating skin penetration. ${ }^{2)}$ The elastic vesicles of deformable liposomes can increase skin permeability. ${ }^{3)}$ In this study, the amount of NaFI in the ED was $1.46 \pm 0.16 \mu \mathrm{g}$, $1.90 \pm 0.60 \mu \mathrm{g}, 1.16 \pm 0.59 \mu \mathrm{g}$, and $6.10 \pm 1.74 \mu \mathrm{g}$ for CLs, PLs, PLs with Tween20, and PLs with $d$-limonene, respectively (Fig. 3). Thus, PLs with Tween 20 as ultradeformable vesicles could penetrate skin better and deliver more NaFI to the receiver compartment than CLs and PLs. Furthermore, the NaFI from PLs with $d$-limonene penetrated ED significantly better than that from other formulations $(p<0.05)$. NaFI deposition was higher in the stratum corneum than in ED for all formulations without $d$-limonene, but PLs with $d$-limonene delivered more NaFI to ED than to the stratum corneum (through the skin to the receiver compartment of the Franz diffusion cell), indicating that $d$-limonene enhances skin penetration. $d$ Limonene can disrupt and dehydrate stratum corneum lipids, resulting in voids and empty spaces in the epidermal region and enhancing drug penetration of skin. ${ }^{28)}$ Some previous reports have discussed the use of ultra-deformable liposomes with skin penetration enhancers. Ultra-deformable liposomes containing Tween 20 as an edge activator and terpenes as skin penetration enhancers have been reported to enhance skin penetration of NaFI. ${ }^{15)}$ Thus, PLs with $d$-limonene penetrated deeper skin layers than other formulations, correlating with the result of skin permeation studies (Fig. 1).

CLSM Study CLSM images were used to visualize skinpenetration depths and the fluorescence intensity of NaFIloaded liposome vesicles and of Rh-PE-probes in phospholipid membranes. In these studies, skin samples were visualized in both nonfollicular and follicular regions for investigation of penetration pathways. As shown in Fig. 4, the fluorescence intensity of free-type NaFI (NaFI solution) was higher at the upper skin layer than NaFI entrapped in liposome formulations. However, the fluorescent compound could penetrate

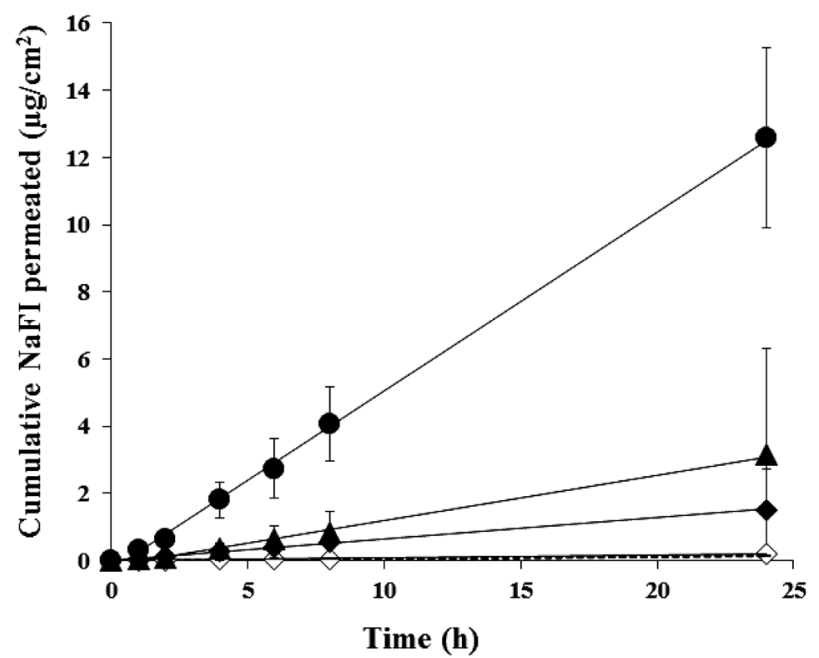

Fig. 2. In Vitro Skin Permeation of NaFI-Loaded Liposome Formulation

Sol $(-($ dot line)), CLs $(\diamond)$, PLs $(\diamond)$, PLs with Tween20 $(\mathbf{\Delta})$, and PLs with $d$ limonene (-) though abdominal porcine skin in Franz diffusion cells at $32 \pm 0.5^{\circ} \mathrm{C}$ Each value represents the mean \pm S.D. $(n=3)$.

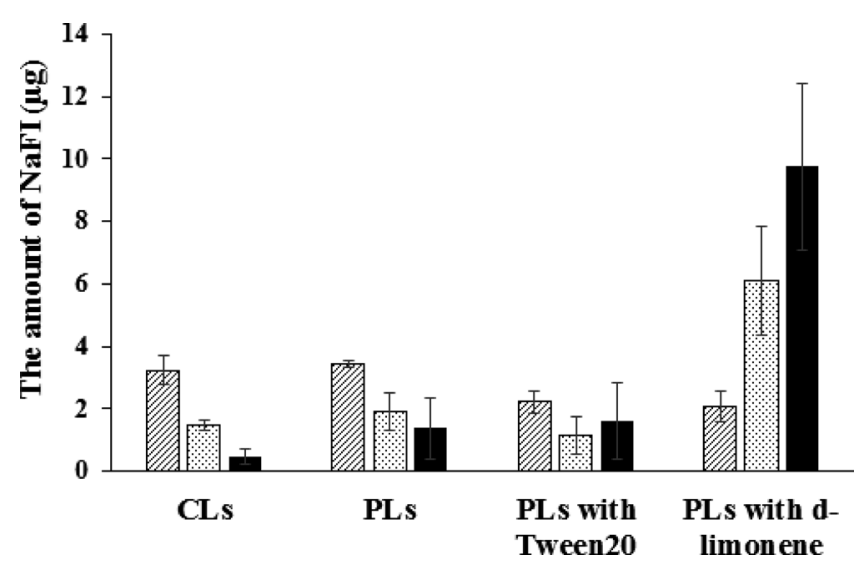

Fig. 3. Comparison of NaFI-Remaining Stratum Corneum (SC, ש), Epidermis and Dermis (ED, B), and Receiver Compartment (ם) from Conventional Liposomes (CLs), PEGylated Liposomes (PLs), PLs with Tween20, and PLs with $d$-Limonene after $8 \mathrm{~h}$ in Vitro Skin Permeation

Each value represents the mean \pm S.D. $(n=3)$.

into deeper skin layers with higher fluorescence intensity at a given depth from PLs with $d$-limonene than from NaFI solution, PLs, and CLs. This was true in both nonfollicular and follicular regions. In nonfollicular regions, PLs with Tween20 or $d$-limonene led to deeper skin penetration and higher NaFI intensity than PLs and CLs. The skin depth of maximum NaFI intensity was $40 \mu \mathrm{m}$ for PLs with $d$-limonene and $50 \mu \mathrm{m}$ for PLs with Tween20, indicating that NaFI was deposited deeper in the skin from PLs with Tween20 than from those with $d$-limonene (Fig. 4A). However, PLs with $d$-limonene gave higher fluorescence intensity than those with Tween20 or those without $d$-limonene. In addition, PLs with $d$-limonene also gave higher Rh-PE intensity than other formulations at the same skin depth (Fig. 4C). In follicular regions, PLs with $d$-limonene gave the highest intensity for NaFI and Rh-PE at a depth of 60 and $55 \mu \mathrm{m}$, respectively (Figs. 4B, D). However, Rh-PE intensity differed little between PLs with $d$-limonene and other formulations (Fig. 4D), indicating that all liposomal formulations led to similar penetration of follicular regions. 

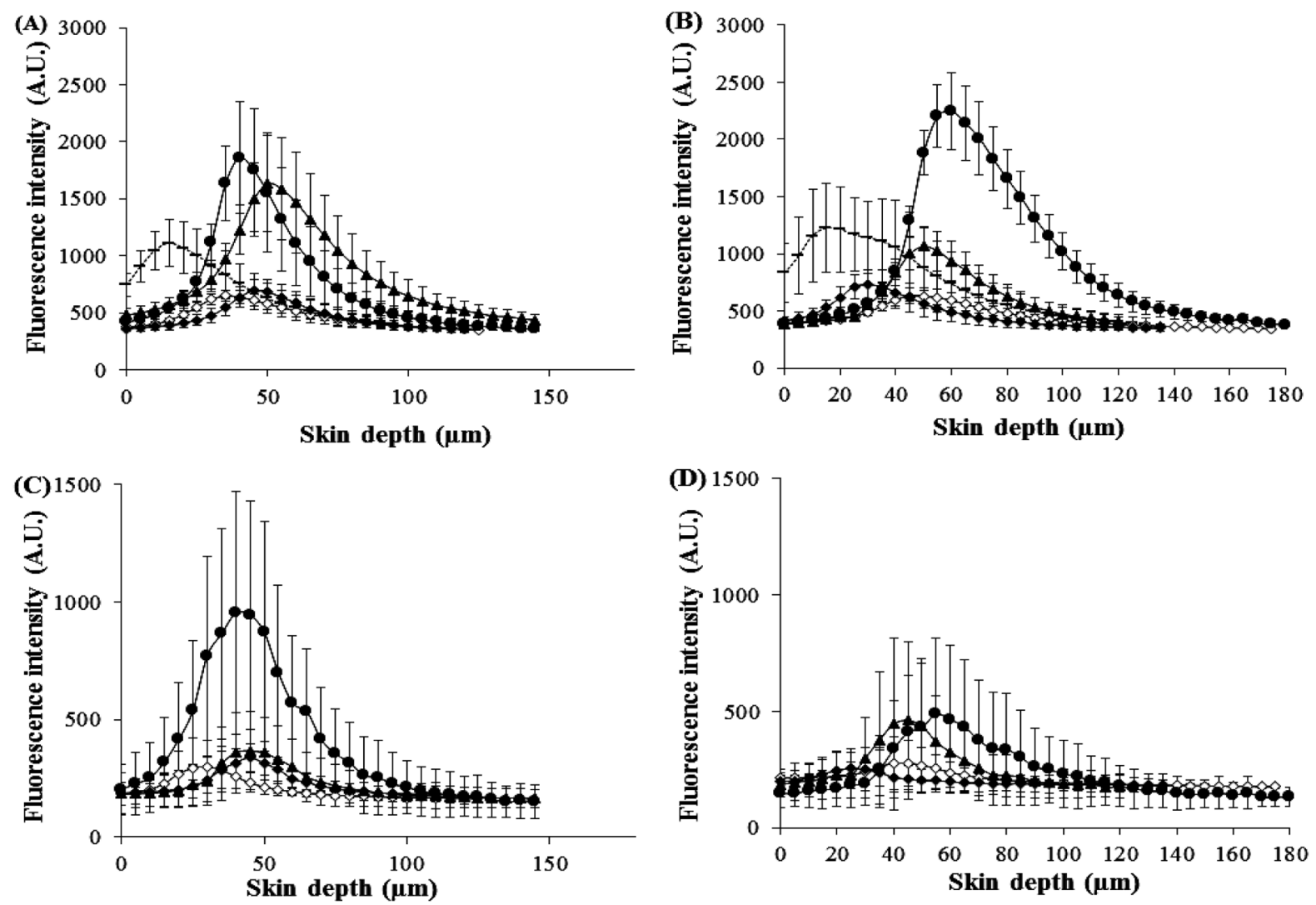

Fig. 4. Fluorescence Intensity Profile of (A) NaFI in Nonfollicular Region, (B) NaFI in Follicular Region, (C) Rh-PE in Nonfollicular Region, and (D) Rh-PE in Follicular Region from Sol (- (Dot Line)), CLs $(\diamond)$, PLs $(\diamond)$, PLs with Tween20 (A), and PLs with $d$-Limonene $(\boldsymbol{\bullet})$

For PLs with $d$-limonene, follicular regions showed higher fluorescence intensity and deeper penetration of NaFI than did nonfollicular regions. However, Rh-PE showed higher fluorescent intensity in nonfollicular than follicular regions (Fig. 4). $\mathrm{Rh}-\mathrm{PE}$ was used as a fluorescence dye to probe phospholipid vesicles. Higher fluorescence intensity of Rh-PE in nonfollicular regions indicates better permeation through the stratum corneum than in follicular regions. PLs with $d$-limonene led to high fluorescence intensity for both NaFI and Rh-PE, suggesting that the phospholipid vesicle and NaFI co-migrated through the stratum corneum. $d$-Limonene in the formulation reduces transfollicular penetration at the expense of transport through the stratum corneum. ${ }^{29)}$ Subongkot et al. $^{6)}$ suggest that ultra-deformable liposome vesicles may attach to some part of the skin (i.e., surface or hair follicles) before releasing the entrapped drug. After release, hydrophilic drugs are transported through pores in the stratum corneum lipid bilayer. In addition to pores, dermal transport of hydrophilic solutes may also occur through hair follicles and sweat ducts: the shunt pathway. ${ }^{30)}$ PLs and CLs showed no difference in the fluorescence intensity of NaFI or Rh-PE between follicular and nonfollicular regions. Deformable liposomes and ethosomes improve skin delivery (permeation and deposition) of the model hydrophilic drug ketotifen (KT) fumarate, with greater improvement of deposition than permeation. ${ }^{31)}$

The $x-z$ axis serial images from CLSM (Fig. 5) show the different penetration depths of NaFI and Rh-PE from PLs with $d$-limonene. In follicular regions, PLs with $d$-limonene penetrated deeper $(180 \mu \mathrm{m})$ than in nonfollicular regions $(145 \mu \mathrm{m})$, indicating a transfollicular pathway for NaFI. The penetration pathway of ultra-deformable liposomes with $d$-limonene was reported to be transfollicular rather than the intercellular or transcellular pathways. ${ }^{12)}$

In this study, three possible mechanisms may improve the skin delivery of drugs by PEGylated liposomes with terpenes. The first mechanism suggests that deformable vesicles can act as drug carriers, whereby intact vesicles enter the stratum corneum carrying vesicle-bound drugs into the skin. The second mechanism suggests that surfactant-based elastic vesicles can act as penetration enhancers, whereby vesicle bilayers enter the stratum corneum and modify intercellular lipid lamellae. This would facilitate penetration of free drug molecules into and across the stratum corneum. ${ }^{4)}$ The third mechanism suggests water-binding PEG molecules can hydrate the skin. In a previous study, PEG layers covered and extended the fluorescence of laurdan (a hydrophilic probe) in the headgroups of $\mathrm{OH}-\mathrm{Chol}$, indicating that the dielectric relaxation times of more highly PEGylated liposomes in water were more hydrated. ${ }^{27)}$ The permeability of skin is affected by changes in the water gradient. Skin permeability increases upon hydration for model drugs with different lipophilicities, which is due to hydration-induced structural alterations in stratum corneum lipids and/or corneocytes. It has also been shown that the relative increase in steady state flux is higher for metronidazole than the more lipophilic methyl salicylate. This difference may be due to swelling of the stratum corneum upon hydration, which reduces the effective solubility of nonpolar drugs in the membrane. ${ }^{32}$

Drug release from vesicles in the stratum corneum is an important step affecting transdermal flux. The rate and amount of released drug is a balance between two factors: 1) drug affinity for vesicles and 2) drug solubility in lipids 


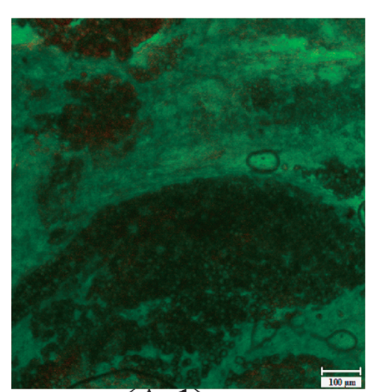

$(\mathrm{A}-1)$

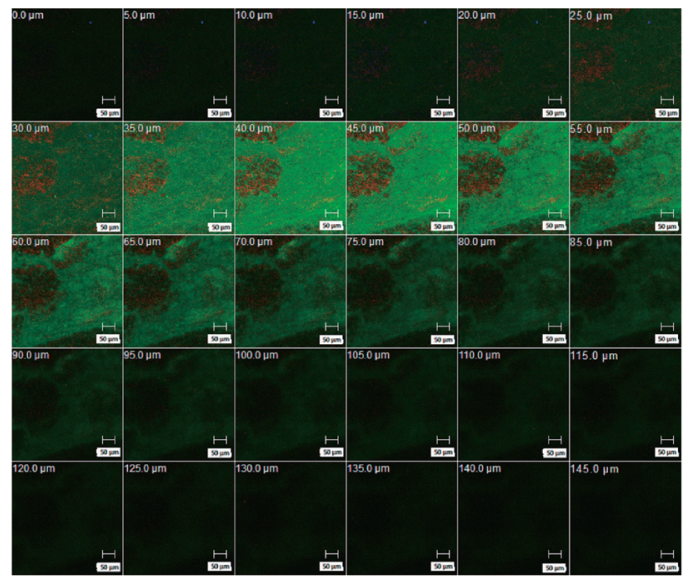

$(\mathrm{A}-2)$

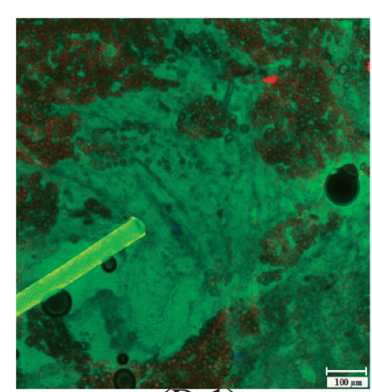

$(\mathrm{B}-1)$

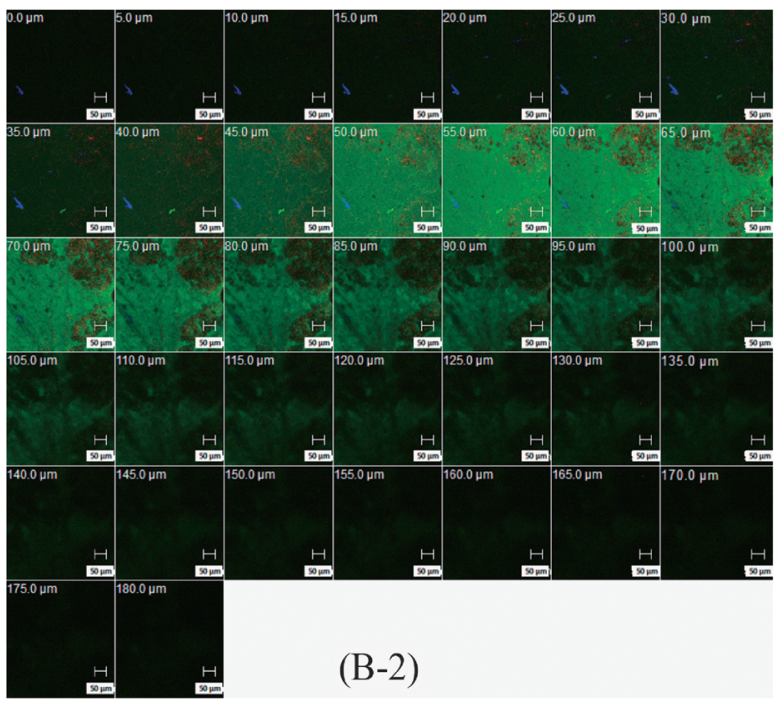

Fig. 5. CLSM Images of Porcine Skin Treated with NaFI-Loaded Rh-PE-Probed PLs with $d$-Limonene at a Time of $4 \mathrm{~h}$ Shows

$x-y$ plane image $(10 \times$ objective lens) at (A-1) nonfollicular region and (B-1) follicular region, and $x-y$ axis serial images (20×objective lens) at (A-2) nonfollicular region and (B-2) follicular region. The images show green fluorescence of NaFI, red fluorescence of Rh-PE, and blue autofluorescence of skin.

of the stratum corneum. Even in a fully hydrated state, the water content in the lowest stratum corneum layers close to the viable epidermis is much lower than in central layers. Therefore, it is expected that, as a result of the osmotic force, vesicles will not penetrate beyond the lowest layers. Thus, drugs must be released from vesicles to reach the circulatory system. ${ }^{4)}$ For hydrophilic compounds, NaFI has higher affinity for PEGylation liposome vesicles than for lipids of the stratum corneum, suggesting that vesicles containing drugs may penetrate the skin and enter circulation. Many researchers have developed PEGylated liposomes to improve blood circulation time by reducing mononuclear phagocyte uptake. However, the application of PEGylated liposomes for delivery of hydrophilic compounds across the skin needs further study in vivo.

\section{CONCLUSION}

Liposomes containing $1 \mathrm{~mol} \% \mathrm{PEG}_{2000}-\mathrm{DSPE}$, Tween 20 and $d$-limonene deliver a significantly increased amount of the hydrophilic NaFI though skin. PEG, a hydrophilic polymer, is grafted onto the surface of the liposomes (PLs), affecting the physicochemical properties and enhancing skin penetration of NaFI. In addition, both Tween20 (an edge activator) and a terpene (as a skin-penetration enhancer) synergistically enhanced penetration. Tape stripping and CLSM revealed skin penetration depths, and drug deposition at each depth, indicating that PLs with $d$-limonene are promising carriers for transdermal delivery of drugs.
Acknowledgments We gratefully thank the Thailand Research Funds through the Royal Golden Jubilee PhD Program (Grant No. PHD/0091/2554) and through the Basic Research Grant (Grant No. 5680016) for financial support. We wish to thank the Division of Medical Molecular Biology (Department of Research and Development, Faculty of Medicine, Siriraj Hospital, Mahidol University) for providing the confocal laser scanning microscope (LSM 510 Meta, Zeiss, Jena, Germany).

\section{REFERENCES}

1) Pierre MB, dos Santos Miranda Costa I. Liposomal systems as drug delivery vehicles for dermal and transdermal applications. Arch. Dermatol. Res., 303, 607-621 (2011).

2) Touitou E, Dayan N, Bergelson L, Godin B, Eliaz M. Ethosomesnovel vesicular carriers for enhanced delivery: characterization and skin penetration properties. J. Control. Release, 65, 403-418 (2000).

3) Cevc G, Blume G. Lipid vesicles penetrate into intact skin owing to the transdermal osmotic gradients and hydration force. Biochim. Biophys. Acta, 1104(1), 226-232 (1992).

4) Honeywell-Nguyen PL, Bouwstra JA. Vesicles as a tool for transdermal and dermal delivery. Drug Discov. Today. Technol., 2, 67-74 (2005).

5) Subongkot $T$, Duangjit $S$, Rojanarata $T$, Opanasopit $P$, Ngawhirunpat T. Ultradeformable liposomes with terpenes for delivery of hydrophilic compound. J. Liposome Res., 22, 254-262 (2012).

6) Subongkot $T$, Wonglertnirant $N$, Songprakhon $P$, Rojanarata $T$, Opanasopit P, Ngawhirunpat T. Visualization of ultradeformable liposomes penetration pathways and their skin interaction by confo- 
cal laser scanning microscopy. Int. J. Pharm., 441, 151-161 (2013).

7) Immordino ML, Dosio F, Cattel L. Stealth liposomes: review of the basic science, rationale, and clinical applications, existing and potential. Int. J. Nanomedicine, 1, 297-315 (2006).

8) Needham D, McIntosch TJ, Lasic DD. Repulsive interactions and mechanical stability of polymer-grafted lipid membranes. Biochim. Biophys. Acta, 1108, 40-48 (1992).

9) Jain S, Tiwary A, Jain N. PEGylated elastic liposomal formulation for lymphatic targeting of zidovudine. Curr. Drug Deliv., 5, 275-281 (2008).

10) Sakai M, Imai $T$, Ohtake H, Azuma H, Otagiri M. Effects of absorption enhancers on the transport of model compounds in Caco-2 cell monolayers: Assessment by confocal laser scanning microscopy. J. Pharm. Sci., 86, 779-785 (1997).

11) Chen M, Liu X, Fahr A. Skin penetration and deposition of carboxyfluorescein and temoporfin from different lipid vesicular systems: In vitro study with finite and infinite dosage application. Int. J. Pharm., 408, 223-234 (2011).

12) Knudsen NØ, Rønholt S, Salte RD, Jorgensen L, Thormann $T$, Basse LH, Hansen J, Frokjaer S, Foged C. Calcipotriol delivery into the skin with PEGylated liposomes. Eur. J. Pharm. Biopharm., 81, 532-539 (2012).

13) Patzelt A, Richter H, Buettemeyer R, Huber H, Blume-Peytavi U, Sterry W, Lademann J. Differential stripping demonstrates a significant reduction of the hair follicle reservoir in vitro compared to in vivo. Eur. J. Pharm. Biopharm., 70, 234-238 (2008).

14) Klang V, Schwarz JC, Lenobel B, Nadj M, Auböck J, Wolzt M, Valenta C. In vitro vs. in vivo tape stripping: Validation of the porcine ear model and penetration assessment of novel sucrose stearate emulsions. Eur. J. Pharm. Biopharm., 80, 604-614 (2012).

15) Kenworthy AK, Simon SA, McIntosh TJ. Structure and phase behavior of lipid suspensions containing phospholipids with covalently attached poly(ethylene glycol). Biophys. J., 68, 1903-1920 (1995).

16) Chain E, Kemp I. The isoelectric points of lecithin and sphingomyelin. Biochem. J., 28, 2052-2055 (1934).

17) Jain S, Jain P, Umamaheshwari RB, Jain NK. Transfersomes-a novel vesicular carrier for enhanced transdermal delivery: development, characterization, and performance evaluation. Drug Dev. Ind. Pharm., 29, 1013-1026 (2003).

18) Maheshwari RGS, Tekade RK, Sharma PA, Darwhekar G, Tyagi A, Patel RP, Jain DK. Ethosomes and ultradeformable liposomes for transdermal delivery of clotrimazole: A comparative assessment. Saudi Pharm. J., 20, 161-170 (2012).

19) Ruozi B, Belletti D, Tombesi A, Tosi G, Bondioli L, Forni F, Vandelli MA. AFM, ESEM, TEM, and CLSM in liposomal char- acterization: a comparative study. Int. J. Nanomedicine, 6, 557-563 (2011).

20) Badran M, Shazly G, El-Badry M. Effect of terpene liposomes on the transdermal delivery of hydrophobic model drug, nimesulide: Characterization, stability and in vitro skin permeation. Afr. J. Pharm. Pharmacol., 6, 3018-3026 (2012).

21) Garbuzenko O, Barenholz Y, Priev A. Effect of grafted PEG on liposome size and on compressibility and packing of lipid bilayer. Chem. Phys. Lipids, 135, 117-129 (2005).

22) Jain AK, Thomas NS, Panchagnula R. Transdermal drug delivery of imipramine hydrochloride. Effect of terpenes. J. Control. Release, 79, 93-101 (2002).

23) Verma DD, Fahr A. Synergistic penetration enhancement effect of ethanol and phospholipids on the topical delivery of cyclosporine. $J$. Control. Release, 97, 55-66 (2004).

24) Oh YK, Kim MY, Shin JY, Kim TW, Yun MO, Yang SJ, Choi SS, Jung WW, Kim JA, Choi HG. Skin permeation of retinol in Tween 20-based deformable liposomes: in-vitro evaluation in human skin and keratinocyte models. J. Pharm. Pharmacol., 58, 161-166 (2006).

25) Escobar-Chávez JJ, Merino-Sanjuán V, López-Cervantes M, UrbanMorlan Z, Piñón-Segundo E, Quintanar-Guerrero D, GanemQuintanar A. The tape-stripping technique as a method for drug quantification in skin. J. Pharm. Pharm. Sci., 11, 104-130 (2008).

26) Maitani Y, Nakamura A, Tanaka T, Aso Y. Hydration of surfactantmodified and PEGylated cationic cholesterol-based liposomes and corresponding lipoplexes by monitoring a fluorescent probe and the dielectric relaxation time. Int. J. Pharm., 427, 372-378 (2012).

27) Lu WC, Chiang B, Huang D, Li P. Skin permeation of $d$-limonenebased nanoemulsions as a transdermal carrier prepared by ultrasonic emulsification. Ultrason. Sonochem., 21, 826-832 (2014).

28) Meidan VM, Bonner MC, Michniak BB. Transfollicular drug delivery-Is it a reality? Int. J. Pharm., 306, 1-14 (2005).

29) Mitragotri S. Modeling skin permeability to hydrophilic and hydrophobic solutes based on four permeation pathways. J. Control. Release, 86, 69-92 (2003).

30) Elsayed MM, Abdallah OY, Naggar VF, Khalafallah NM. Deformable liposomes and ethosomes: Mechanism of enhanced skin delivery. Int. J. Pharm., 322, 60-66 (2006)

31) Björklund S, Engblom J, Thuresson K, Sparr E. A water gradient can be used to regulate drug transport across skin. J. Control. Release, 143, 191-200 (2010).

32) Varman RM, Singh S. Investigation of effects of terpene skin penetration enhancers on stability and biological activity of lysozyme. AAPS PharmSciTech, 13, 1084-1090 (2012). 